\title{
Diagnostic imaging in the diagnosis of acute complications of bariatric surgery
}

\author{
Antonio Catelli ${ }^{1 A, B, C, D, E, F}$, Antonio Corvino ${ }^{2 A, B, C, C, E, F,}$, Giovanni Loiudice ${ }^{1 A, B, C, D, E, F}$, Anna Tucci ${ }^{1 A, B, C, D, E, F}$, \\ Mario Quarantelli ${ }^{1 A}$, Pietro Venetucci ${ }^{1 A, B, C, D, E, F}$ \\ ${ }^{1}$ Advanced Biomedical Sciences Department, University Federico II of Naples (UNINA), Naples, Italy \\ 2Motor Science and Wellness Department, University of Naples "Parthenope", Naples, Italy
}

\section{Abstract}

Purpose: The aim of study is to identify the frequency of acute complications and imaging findings at gastro-intestinal transit (GI) and computerised tomography (CT) in a group of obese patients who developed clinical suspicion of acute complications (painful and meteoric abdomen, nausea, vomiting, fever, intestinal blockage) in post bariatric surgery.

Material and methods: We retrospectively review 954 obese patients who underwent bariatric surgery between 2013 and 2019. The study included 72 patients who developed clinical suspicion of acute complications (painful and meteoric abdomen, nausea, vomiting, fever, intestinal blockage) within 6 days of bariatric surgery of sleeve gastrectomy, gastric banding, gastric bypass with Roux loop confirmed by CT, and who underwent a gastrointestinal transit before the CT examination.

Results: GI exam allowed visualisation of $58 \%$ of complications. Analysing the data for each surgical technique, 46 post-operative complications were found involve gastric banding. The most frequent was bandage migration (26 cases, $56 \%$ ), identified in all cases at GI transit and then confirmed on CT.

Conclusions: The study suggests that CT should be used to clarify all doubtful or clinically discordant GI transit exam results. The participation of a radiologist in qualification and post-operative evaluation is important for bariatric surgery patients.

Key words: bariatric surgery, CT, sleeve gastrectomy, post-surgical complications, GI transit X-ray, BMI.

\section{Introduction}

Obesity is a chronic, evolutionary and recurrent pathological condition with complex aetiopathogenesis, characterised by excess weight due to the accumulation of body fat, which worsens the quality of life and causes complications that can lead to death.

The degree of obesity is defined on the basis of a score, the body mass index (BMI), which obtained by calculating the weight in kilograms $(\mathrm{kg})$ divided by the square of the height in metres $\left(\mathrm{m}^{2}\right)$. The term overweight indicates adults with $\mathrm{BMI} \geq 25 \mathrm{~kg} / \mathrm{m}^{2}$, although some authors refer only to subjects with BMI between 25 and $29.9 \mathrm{~kg} / \mathrm{m}^{2}$. Preobesity is defined by a BMI between 25 and $29.9 \mathrm{~kg} / \mathrm{m}^{2}$. Obesity is defined by a BMI $\geq 30 \mathrm{~kg} / \mathrm{m}^{2}$ [1-3]. However, $\mathrm{BMI}$ is an approximate indicator because body weight is not only influenced by fat mass but also by lean mass. The methods available to calculate the fat mass are the following: the count of skin folds, the evaluation of their thickness (plicometry), bioimpedance (optional increasingly required in common scales), or using techniques (magnetic resonance, hydrostatic weighing, computerised tomography [CT], etc.) [1-3]. In cases of non-response to medical treatment or in the presence of severe obesity,

Correspondence address:

Antonio Corvino, Motor Science and Wellness Department, University of Naples "Parthenope", via F. Acton 38, I-80133 Naples, Italy, e-mail: an.cor@hotmail.it

Authors' contribution:

A Study design · B Data collection · C Statistical analysis · D Data interpretation · E Manuscript preparation · F Literature search · G Funds collection 
the alternative is bariatric surgery. The guidelines recommended and adopted by the Italian Society of Obesity Surgery identify, as a first step, that of carrying out a psychodiagnostic evaluation aimed at identifying not only the personality characteristics of the patient but also any specific contraindications such as the state anxiety and depression for bariatric surgery [1-3].

\section{Bariatric surgery}

Bariatric surgery has emerged in recent years as an optimal therapeutic strategy, especially in severe obesity, when medical therapy based mainly on diet fails. Its notable progress lies mainly in the possibility of performing operations laparoscopically. This minimally invasive technique reduces hospitalization times, with a positive impact on health care costs, and reduces post-operative pain and complications related to surgical wounds [4-6].

\section{Surgical techniques}

Bariatric surgery includes several surgical techniques, which can be classified as restrictive or malabsorbent. The former are based on the volumetric reduction of the stomach and therefore on the reduction in food and calorie intake, without a significant impact on the absorption of essential nutrients. They include gastric banding, sleeve gastrectomy, and gastric bypass with Roux loop packaging. The latter limit the absorption by resection and bypass of intestinal tracts; among these, the most performed intervention is biliopancreatic diversion with duodenal switch [7]. For each technique, the advantage translates into a significant weight loss, with a consequent improvement in quality of life, but also in a clear reduction of comorbidities and metabolic disorders, with prolongation of survival [8].

\section{Endoscopic procedures}

These include an endogastric balloon packed in silicone, which is used orally or under endoscopic guidance, and the EndoBarrier, which is an endoscopic implantable disposable device consisting of a waterproof fluoropolymer tube about $60 \mathrm{~cm}$ long; it is positioned in the duodenal bulb so as to exclude the duodenum and the first part of the fast from the food transit.

\section{Diagnostic imaging}

Diagnostic imaging plays an important role [9-12] because the number of bariatric surgeries is increasing, which has caused an increase also in the number of radiological investigations performed for the study of obese patients, both in the phases prior to treatment and in the post-operative period. For this reason, it is important that radiologists are familiar with the post-operative anatomy derived from the main surgical techniques and possible complications [13]. The most performed post-operative radiological investigations are radiography of the abdomen followed by the study of gastro-intestinal transit (GI) through administration of water-soluble oral contrast and CT. The GI transit study offers a limited overview; it generally allows a rapid assessment of the presence of free subdiaphragmatic air and the correct position of the gastric banding. In addition, it can directly identify the presence of anastomotic dehiscence both in the sleeve gastrectomy and in the by-pass, and indirectly suggests possible obstructive complications, for which CT deepening is used [14]. Instead, in the case of the absence of abnormal findings and good clinical conditions, the patients can have the nose gastric tube removed and they can be discharged. In the presence of doubtful findings or possible complications, a CT scan is performed. Due to its high panoramic nature, CT is the ideal examination for the evaluation of post-operative complications, especially if obstructive syndromes, internal hernias, or abscess collections are suspected [15].

\section{Acute complications of bariatric operation}

Bariatric procedures are generally safe and effective, but they can be associated with devastating complications, some of which can be fatal if not addressed quickly. Acute complications from bariatric surgery generally occur 6 days after surgery, and they include leakage, stenosis, bleeding and venous thromboembolic events (VTE), acute appendicitis, acute diverticulitis, acute pancreatitis, and gallstone disease and band migration (gastric bending) [16].

\section{Material and methods}

\section{Population}

We retrospectively review 954 obese patients $(744 \mathrm{fe}-$ males, $78 \%$; 210 males, 22\%) aged between 18 and 65 years (mean age 43 years) who underwent bariatric surgery from 2013 to 2019. In particular, 269 patients underwent laparoscopic gastric banding, 525 patients with sleeve gastrectomy, and 160 patients with gastric bypass with Roux loop. The study included 72 patients who developed clinical suspicion of acute complications (painful and meteoric abdomen, nausea, vomiting, fever, intestinal blockage) within 6 days of bariatric surgery of sleeve gastrectomy, gastric banding, gastric bypass with Roux loop confirmed by $\mathrm{CT}$, and who underwent a gastrointestinal transit before the $\mathrm{CT}$ examination. In particular, 42 patients confirmed the complication of the gastrointestinal transit exam. The remaining 30 patients had a negative gastrointestinal transit examination. All patients of the study underwent a GI exam $24 \mathrm{~h}$ after the onset of clinical symptoms of suspected acute surgical complication. The CT scan was performed $24 \mathrm{~h}$ after the GI exam. 


\section{Imaging techniques}

All included patients underwent GI transit control before and after oral administration of contrast agent (Gastrografin, $80 \mathrm{ml}$ ). The GI transit check was performed with (Figure 1) Rx of the abdomen in white in AP orthostasis. First radiogram after contrast medium per os: oesophagus-gastric region - AP projection in orthostasis. Second radiogram: gastro-duodenal region left anterior oblique projection (OAS) with right hip supported. Third radiogram: jejunoileal region AP panoramic projection.

The equipment used was a 64 -channel multi-detector CT. The initial scan was performed after filling the GI tract with positive oral contrast and was followed by intravenous administration of $120 / 160 \mathrm{ml}$ of iodinated contrast medium with acquisitions, using the "bolustracking" technique in the arterial, portal, and in some patients even late.

\section{Results}

Overall, among all surgical interventions, the most frequent complication was obstructive syndrome of the small intestine (small bowel obstruction - SBO), for a total of
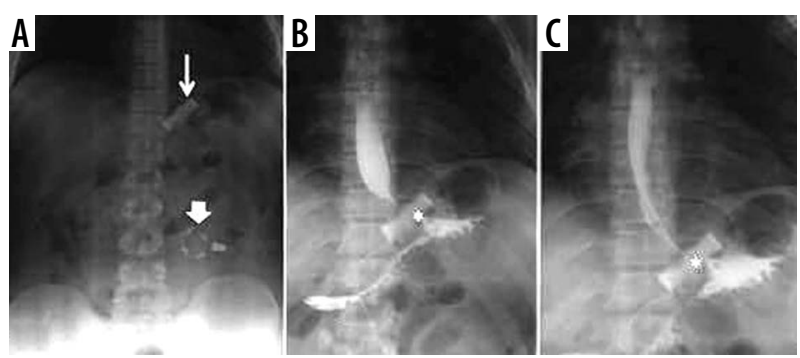

Figure 1. Gastro-intestinal transit in a patient with a gastric band. A) Thin arrow: gastric band ring; thick arrow: external reservoir. B, C) Asterisk: regular passage through the bandage of contrast medium per os

27 cases (38\% of all complications). In this heterogeneous group of pathologies, occlusions from anastomotic stenosis $10 / 27$ (37\%), dolicho-catheter $2 / 27$ (7\%), flies $2 / 27$ (7\%), strangulated laparoceles $8 / 27(30 \%)$, and internal hernias 5/27 (19\%) were included. The second most frequent complication was bandage migration ( 26 cases, $36 \%$ of all complications), which only concerned gastric band surgery (Figures 1-4); simple and poorly positioned (intravisceral and extra-visceral) migrations were considered. The other complications encountered were anastomotic dehiscence (16 cases, $22 \%$ of all complications) and haemorrhage (3 cases of non-replenished haematomas,
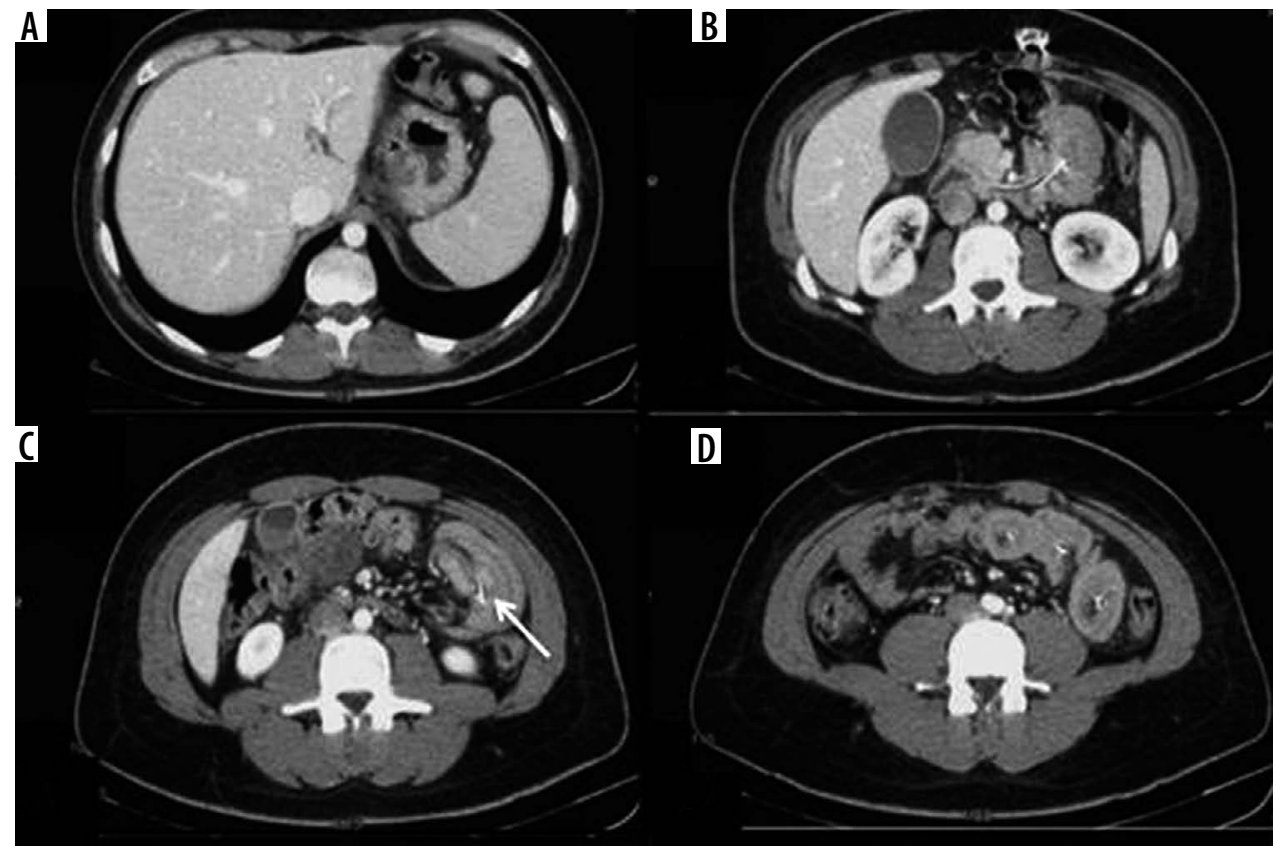

E

\section{F}

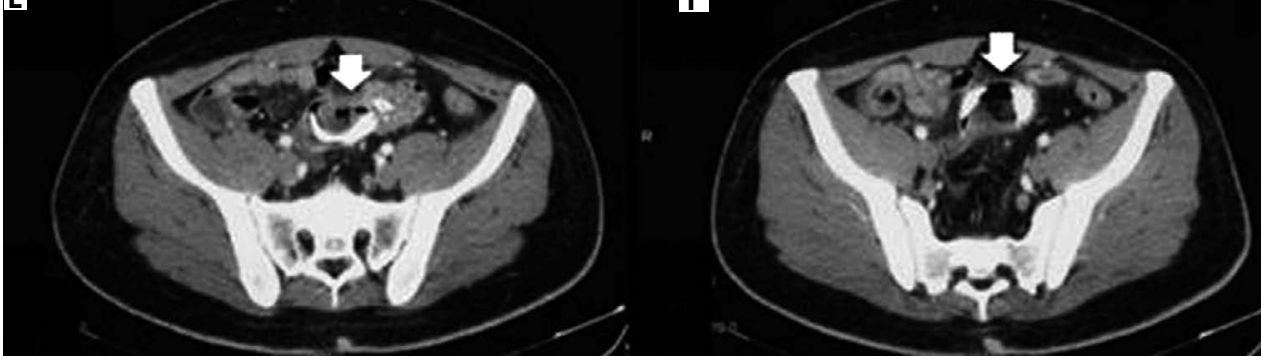

Figure 2. Gastric band intra-ileal migration. A) Absence of bend in the typical site. B-D) Thin arrow: the presence of the connection catheter between the reservoir and the gastric band ring is highlighted. E, F) Bandage ring migrated to the endo-luminal ileal site (thick arrow) 


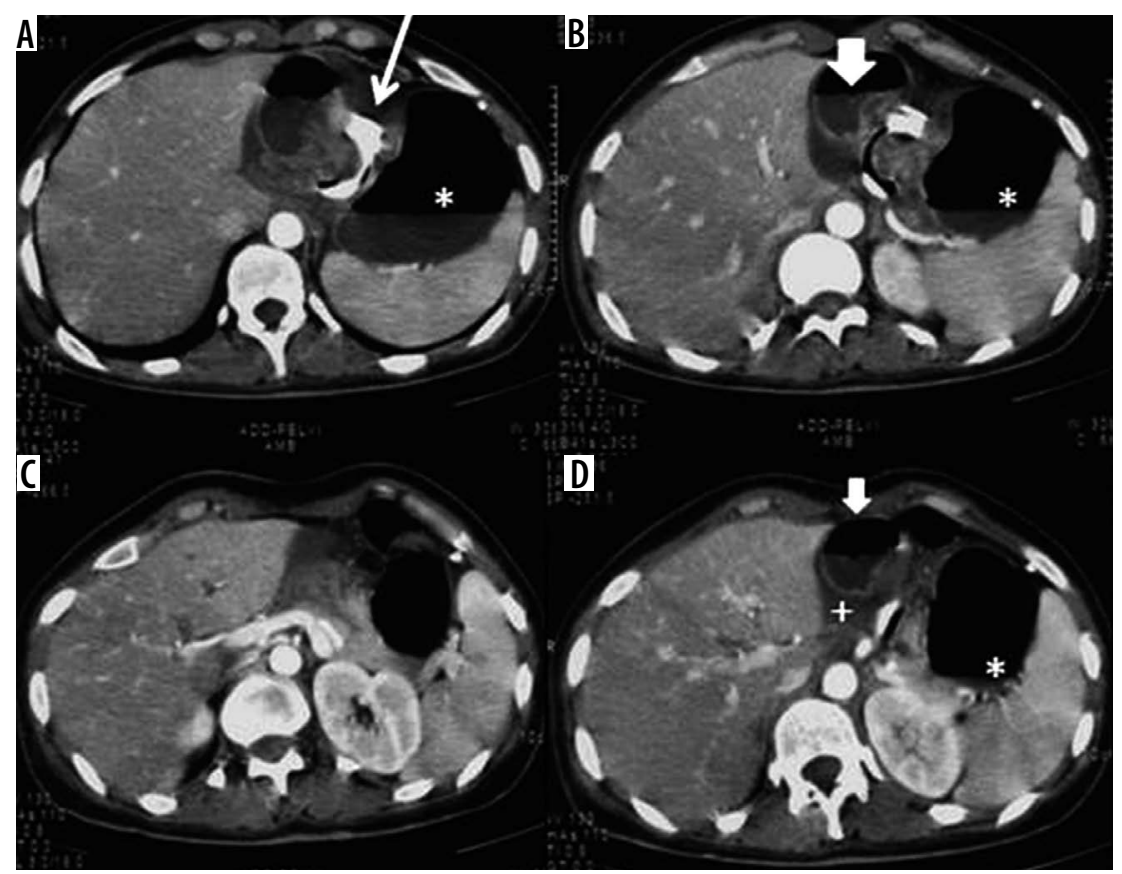

Figure 3. Gastric volvulus in a patient with gastric banding. Over-distended gastric bottom with fluid stagnation (asterisk) and gastric band ring (thin arrow). Remaining gastric portion rotated and suffering with perivisceral fluid (thick arrow) and perivisceral liquid (+)
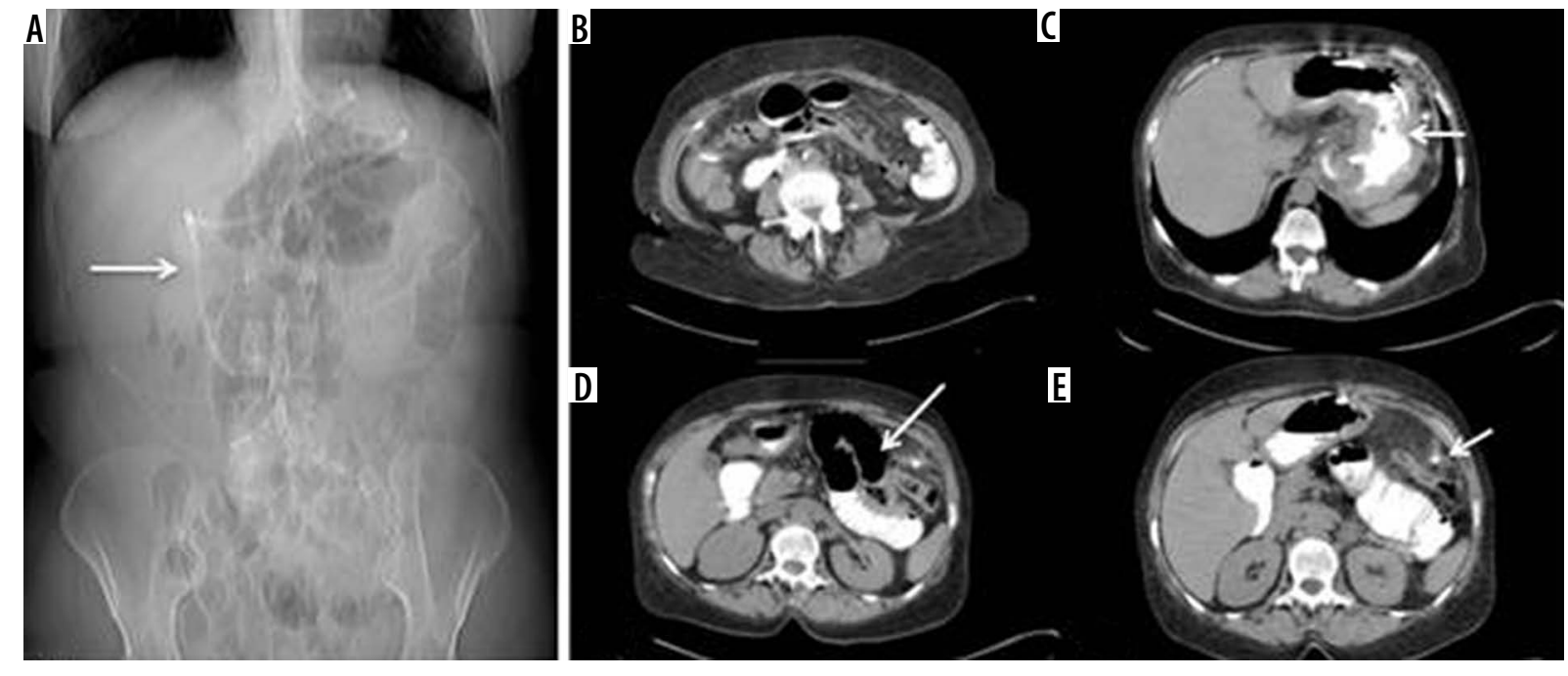

E

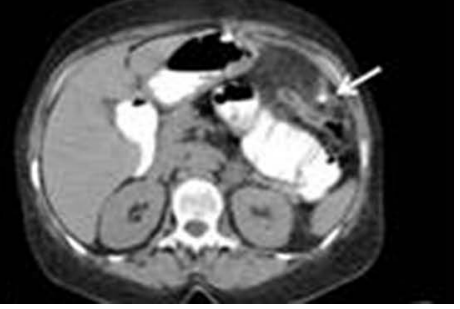

Figure 4. Intestinal obstruction from a gastric band catheter. A) Scout-view: abnormal and elongated course of the bandage device catheter. B) Compression of the jejunal loop secondary to traction exerted by an adipose tissue band attracted by the catheter in an abnormal location. C, D) Over-distension of the stomach, duodenum, and fasting. E) Traction on the parietal peritoneum

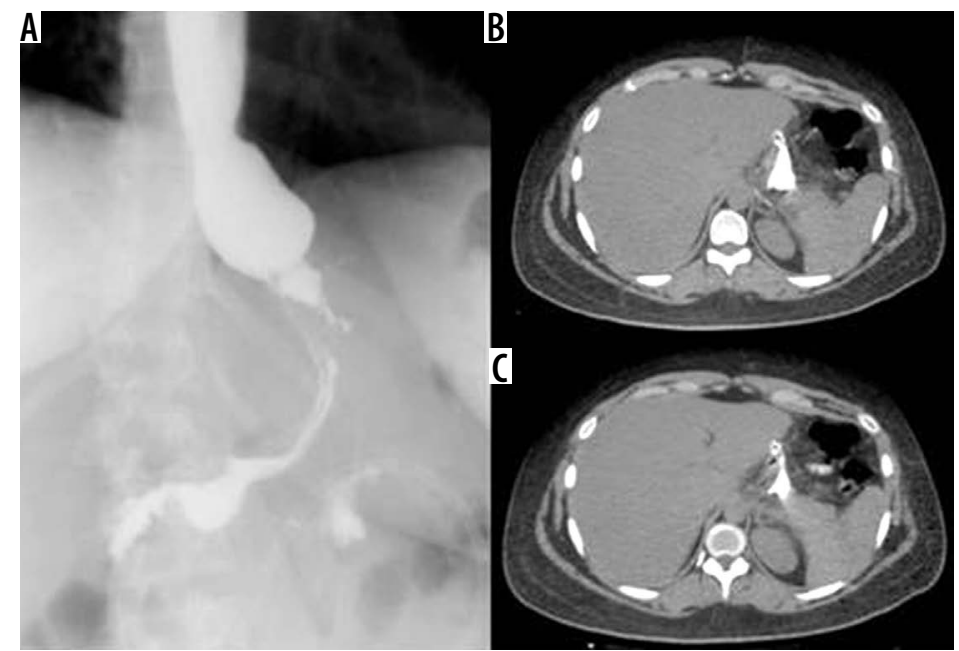

Figure 5. Sleeve gastrectomy suture dehiscence. Extravasation of contrast medium from the proximal region of the suture, demonstrated both during the Gl transit and in CT 


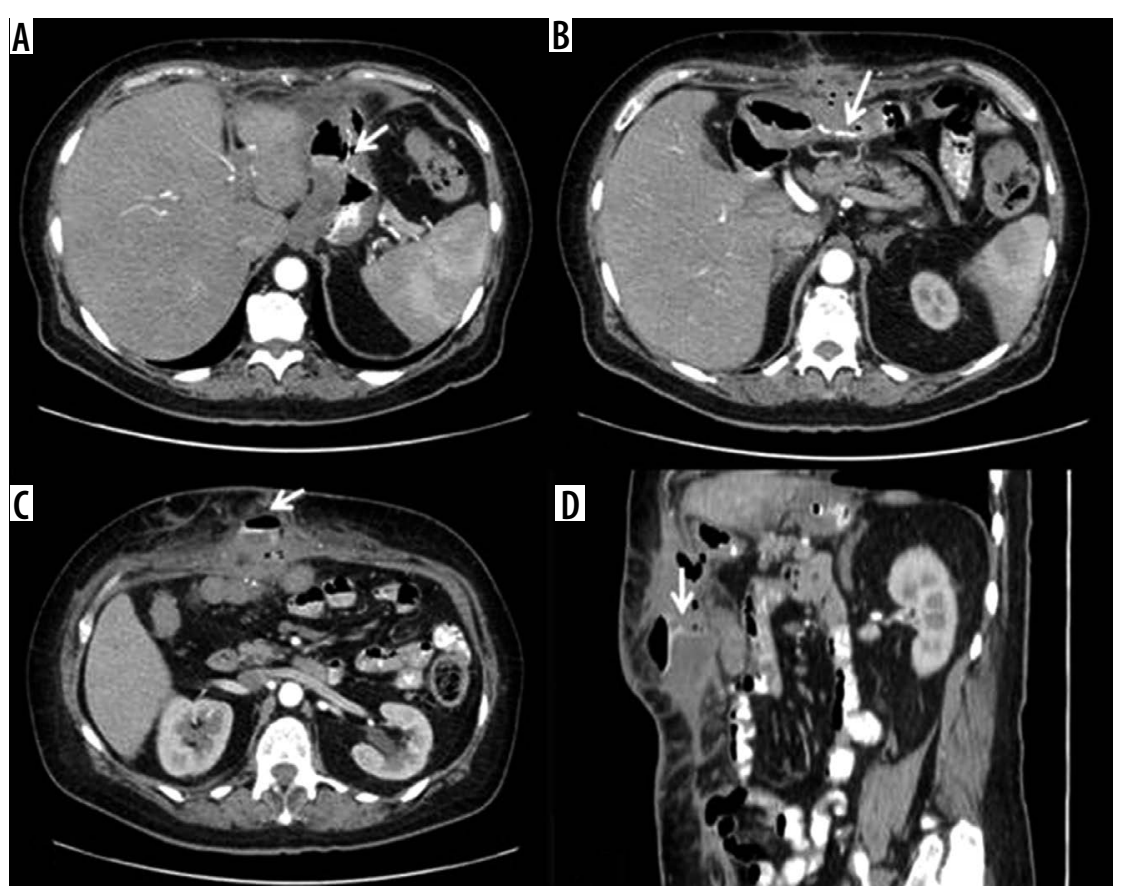

Figure 6. Anastomotic dehiscence in outcome of sleeve gastrectomy with fistulised collection at the level of the anterior abdominal wall. A) Via fistula with the stomach. B-D) Route of the fistula in axial and sagittal oblique section. C) Opacification of the collection under fascial
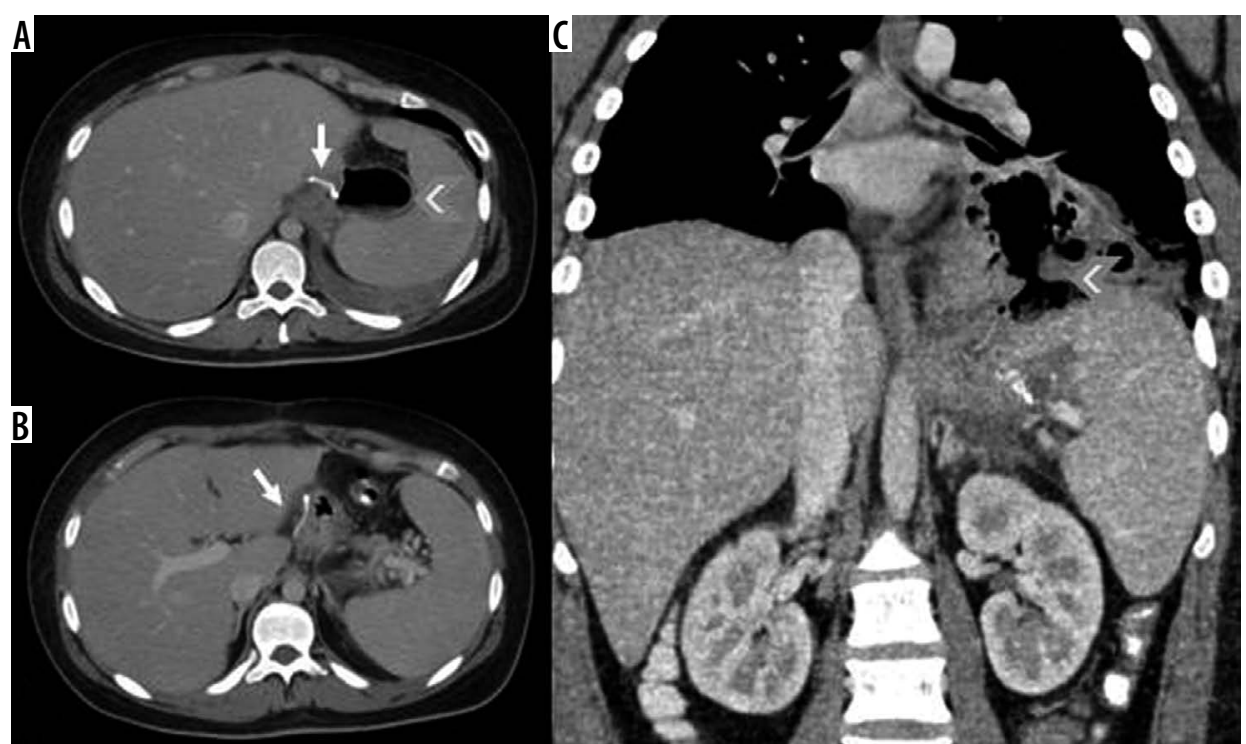

Figure 7. Anastomotic suture dehiscence in sleeve gastrectomy outcomes with transdiaphragmatic fistulised lung collection. A, B) Metallic sutures in sleeve gastrectomy outcomes (white arrow), with adjacent hydro-aerial collection (arrowhead). C) Transdiaphragmatic fistula route (arrowhead) with abscess collection in the lung parenchyma

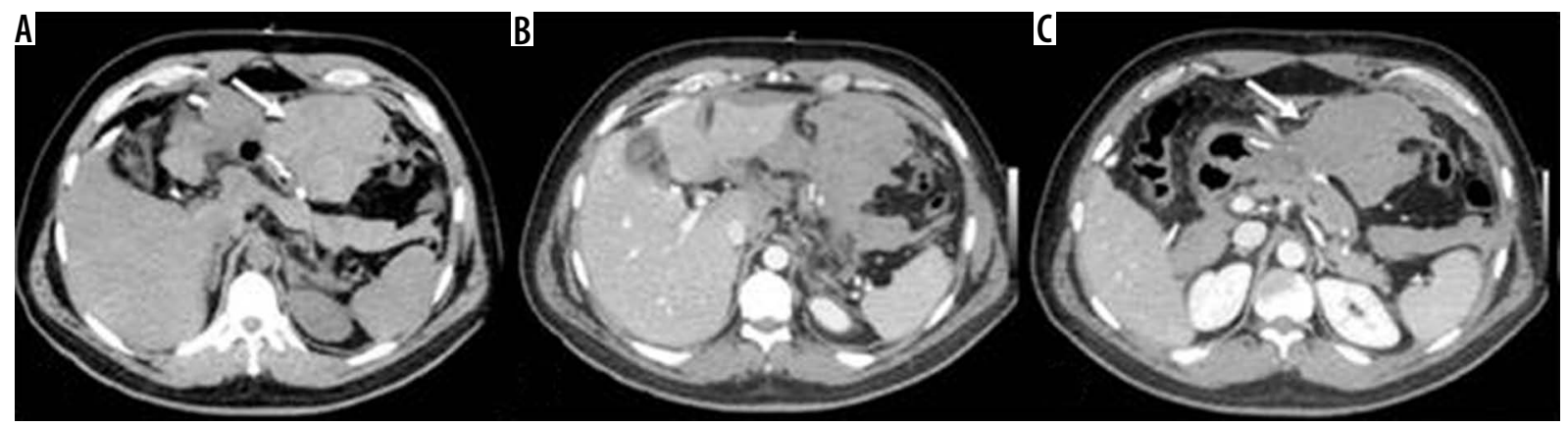

Figure 8. Intraperitoneal haematoma adjacent to the sleeve gastrectomy suture. A) Spontaneous hyperdense haematoma in the pre-contrast phase in the $2 / 3$ distal of the gastric suture. $B, C$ ) In the post-contrast phases an active supply cannot be documented 


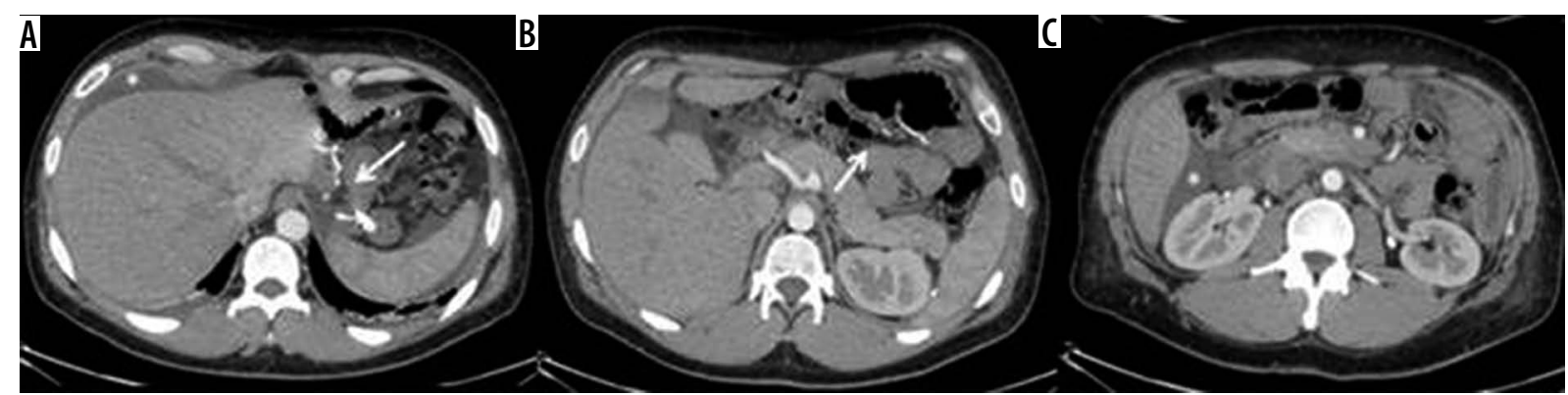

Figure 9. Internal hernia in a patient with a gastric bypass. A) Arrow: gastric bypass results - asterisk: perihepatic liquid flap. B) Thickened peri-anastomotic fat (arrow). C) Fluid stratum in Morrison (asterisk)
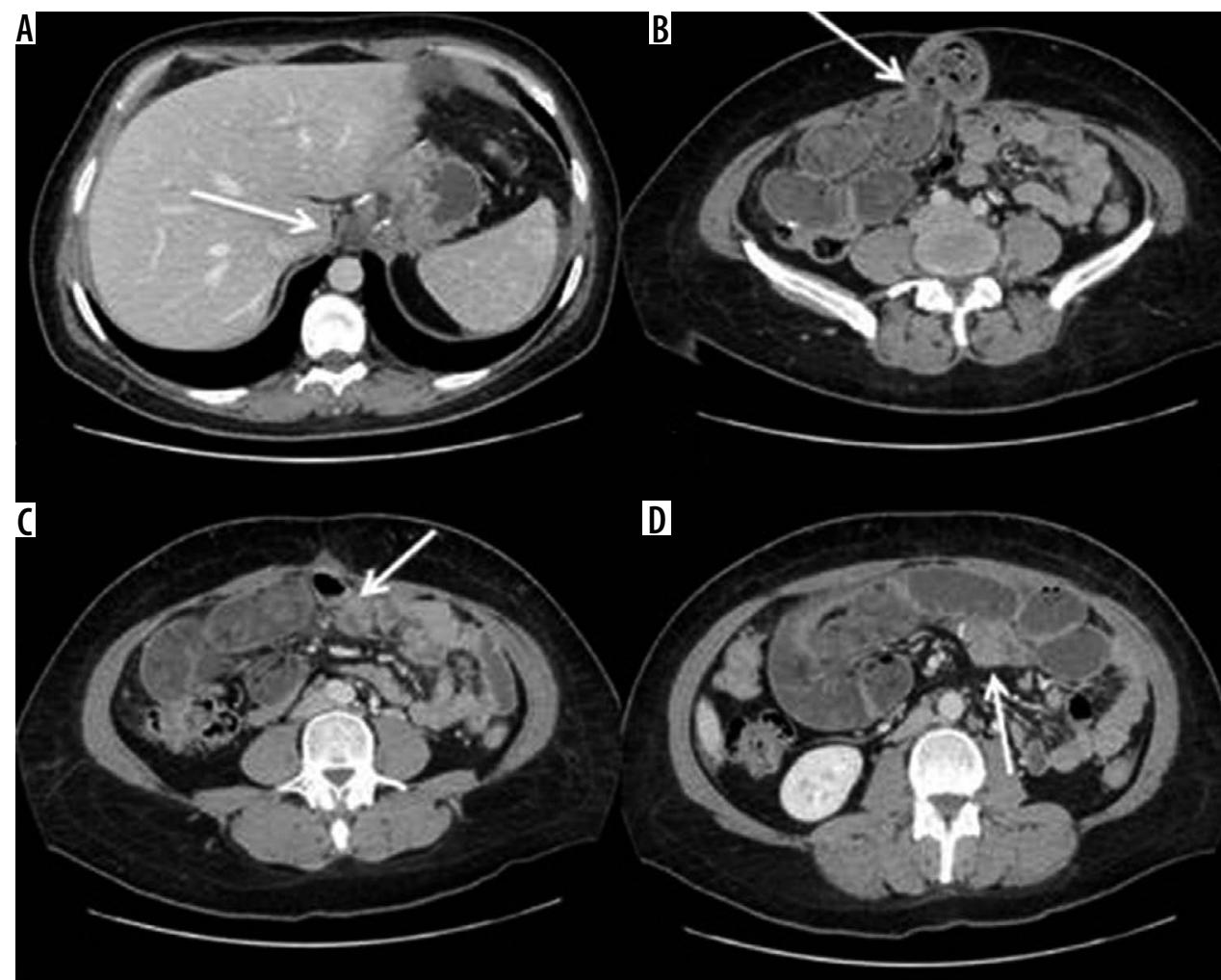

Figure 10. Occlusion on the bridle at the entero-enteric anastomosis in a patient operated on by gastric bypass. A) Outcomes of gastric bypass surgery (arrow). B) Laparocele input. C) Laparocele output. D) Entero-enteric anastomosis loop foot housing the mechanical stop on the bridle (arrow)
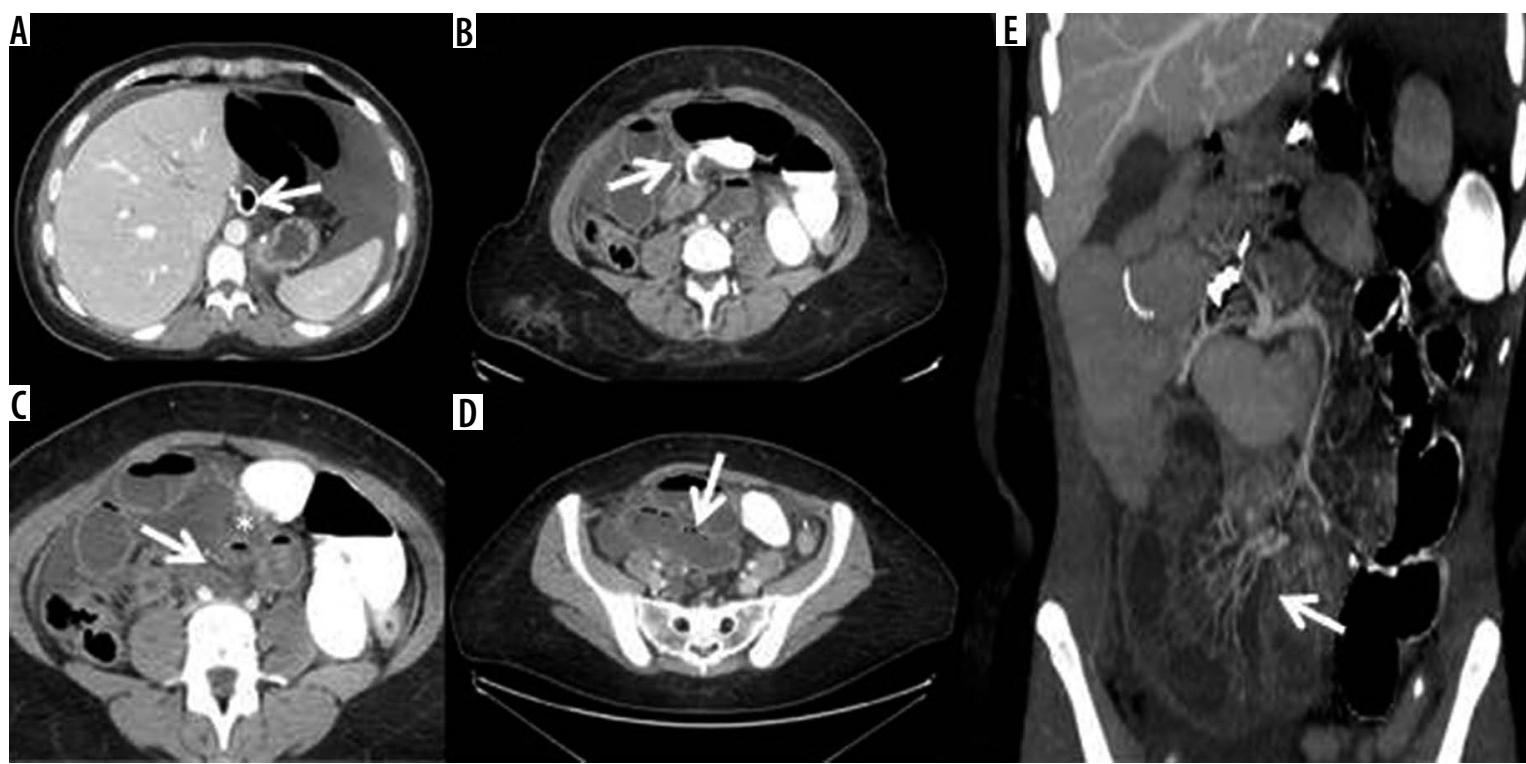

D
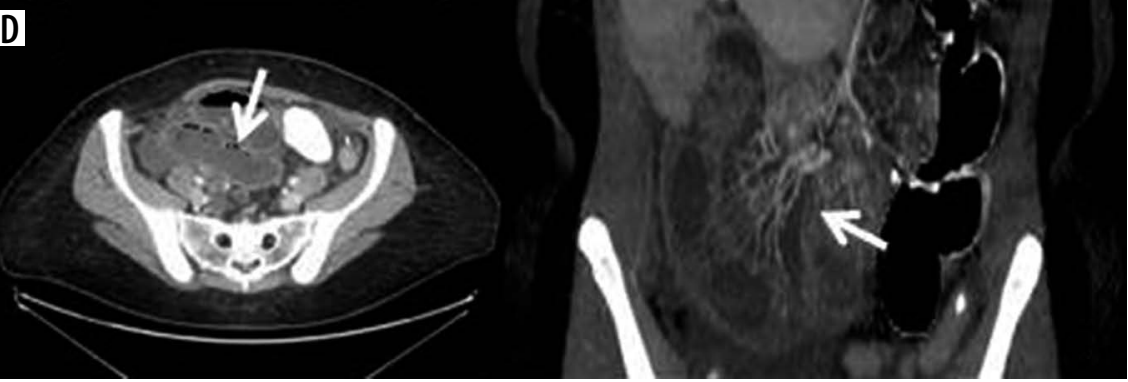

Figure 11. Volvulus in patient operated by gastric by-pass. A) Outcomes of gastric by-pass. B) Volvulus entrance. C) Volvulus exit (arrow) and suffering loop (asterisk). D) Faecaloid stasis. E) Reconstruction on the coronal plane shows signs of venous hypertension in suffering loops with stop of the venous vessels 
Table 1. Schematic illustration of the surgical complications frequency and correlation with the imaging examinations

\begin{tabular}{|l|c|c|c|c|}
\hline \multirow{3}{*}{ Kind of surgery } & \multicolumn{2}{|c|}{ Complications } & UGI & CT \\
\hline \multirow{3}{*}{ Gastric bandage } & TOT & $46(17 \%)$ & $33(72 \%)$ & $46(100 \%)$ \\
\cline { 2 - 5 } & MB & $26(56 \%)$ & $26(100 \%)$ & $26(100 \%)$ \\
\cline { 2 - 5 } & SBO & $20(44 \%)$ & $7(35 \%)$ & $20(100 \%)$ \\
\cline { 2 - 5 } & TOT & $16(22 \%)$ & $6(37 \%)$ & $16(100 \%)$ \\
\cline { 2 - 5 } & LEAK & $13(81 \%)$ & $6(46 \%)$ & $13(100 \%)$ \\
\hline \multirow{3}{*}{ Gastric by-pass } & EMG & $3(19 \%)$ & $0(0)$ & $3(100 \%)$ \\
\cline { 2 - 5 } & TOT & $10(14 \%)$ & $3(30 \%)$ & $10(100 \%)$ \\
\cline { 2 - 5 } & LEAK & $7(30 \%)$ & $1(33 \%)$ & $3(100 \%)$ \\
\hline \multirow{2}{*}{ Total } & SBO & $2(29 \%)$ & $7(100 \%)$ \\
\hline
\end{tabular}

UGI - gastrointestinal transit, MB - bandage migration, SBO - occlusive syndrome of the small intestine, LEAK - anastomotic dehiscence, EMG - bleedw

$4 \%$ of all complications). Analysing the data for each surgical technique, it emerges that of the 46 patients undergoing gastric banding complications, the greatest of which was bandage migration ( 26 cases, 56\%), all were identified in GI transit and confirmed by CT, followed by 20 obstructive syndromes of the small intestine (44\%), 7 of which identified in GI transit and all confirmed by CT. Of the 16 patients who underwent sleeve gastrectomy complication (22\% of all complications) (Figures 5-8), in particular, 13 cases of suture dehiscence were found ( $81 \%$, 6 of which were identified in GI transit) and 3 cases of internal bleeding ( $1 \%$, none of which identified in GI transit). Of the 10 patients who underwent gastric bypass with Roux loop complication (14\%) (Figures 9-11), 3 (30\%) anastomotic dehiscence (only one identified at GI transit) and 7 (70\%) obstructive syndromes of the small intestine were subsequently confirmed by CT (Table 1). 50/72 (69\%) patients underwent surgery. Intraoperative confirmation occurred for 50/50 (100\%) patients treated. 4/50 (4\%) patients underwent further surgical treatment.

\section{Discussion}

Analysing the data for each surgical technique, 46 postoperative complications were found due to gastric banding. The most frequent was bandage migration (26 cases, $56 \%$ ), identified in all cases at GI transit and then confirmed on CT. Bandage migrations can be divided into simple, in which the distal dislocation occurs in the extravisceral, and complex, with intra-visceral migration. In the first case, the clinic is rather silent, and it is sufficient to perform an X-ray of the abdomen to identify the relocation of the bandage, which generally takes place at the gastric body level, with herniation of the bottom through the bandage. It tends to assume a horizontal position, in which the front and back do not match, producing an $\mathrm{O}$-shaped image, also called "O-shape sign", which is highly suggestive of bandage migration $[17,18]$. Treatment can be postponed and is typically based on simple removal. For this complication, the GI transit has proven to be an optimal technique for the rapid identification of the bandage relocation with high sensitivity (100\%). In complex migrations the clinical presentation is more critical because it presupposes erosion and perforation of the affected bowel [19]. For this reason, CT is used to identify the intra-visceral site of the bandage and the associated additional complications. The data of our case studies are in accordance with those of the literature, which reports bandage migration rates ranging from 4 to $13 \%$ [20]. Furthermore, of the 26 migrations found, only $1(<1 \%)$ was found to be intra-visceral, with dislocation at the distal ileum level; this finding is also in line with that found in similar cases [21]. The second most frequent complication in patients undergoing gastric banding was intestinal occlusion (44\%). Of these, 7 were preliminarily identified on GI transit and all subsequently confirmed on CT. In one case, the occlusion was determined by the twisting of the stomach with the fulcrum on the bandage, which caused a volvulus, with consequent suffering of the walls of the bowel. The cause is probably attributable to incorrect positioning and adjustment of the device; however, it may also be secondary to gastric tissue laxity incorrectly assessed in the intra- and perioperative phase. In this case the CT was fundamental for accurate confirmation of the diagnosis, also providing an urgency criterion based on the characteristics of the vascularisation of the gastric walls. The course and length of the connection catheter between the external reservoir and the bandage ring must be carefully evaluated during the positioning phase; in fact, in our experience, we can count an occlusive syndrome secondary to abnormal traction effects that an overly long catheter had generated on a jejunum-ileal segment. Direct abdominal $\mathrm{X}$-ray is an aid to evaluate any painful appearance and an abnormal course of the catheter. However, CT with contrast medium was decisive in accurately locating both the occlusion site and the presence of any signs of loop pain. This complication is specifically related to the gas- 
tric band surgery. Also, for this type of complication, the data we found are in accordance with those reported in the literature, although most reports consider gastric volvulus separately, compared to other occlusive syndromes $[16,18,19]$. Overall, for the evaluation of gastric band complications, GI transit represents a reliable method for identifying early complications related to malpositioning and late from distal migration, while it has a more limited role in occlusive syndromes. In all cases, CT was decisive in the best characterisation of the pathology. In our case studies, sleeve gastrectomy was the surgery with the lowest complication rate. This finding can be ascribed in the first hypothesis to a less invasive technique, characterised by a significant saving in the physiological course of the alimentary canal and by the absence of surgical anastomosis. In accordance with the literature data, the most frequent complication was suture dehiscence, specifically related to the bariatric surgical act $[16,22,23]$. The consequences of a dehiscence can be manifold, ranging from simple extra-luminal extravasation of gastric material to the formation of intra-abdominal collections, trans-parietal fistulas, as well as trans-diaphragmatic fistulas with consequent involvement of the pulmonary parenchyma. At the preliminary X-ray examination, after administration per os of water-soluble iodinated contrast (Gastrografin), it is possible to appreciate the presence of subtle fistulosis through the surgical scar; subsequently, a diagnostic examination with a CT examination may be appropriate to assess the fistulous path more accurately, as well as the presence of collections and their possible supply. The therapy of anastomotic dehiscence provides, if the patient's clinical condition permits, a first conservative approach, positioning a nasogastric suction tube, with the aim of reducing the endo-luminal pressure and removing the gastric secretion to favour the processes of scarring. This approach was decisive in only 1 case of anastomotic dehiscence found by us. A subsequent CT scan with contrast medium by mouth can better evaluate the presence of extra-luminal spills and suggest the choice of a possible positioning of endoscopic gastric stent or repackaging of the gastric suture. It may also be necessary to drain circumscribed collections also under CT guidance.

The remaining complications are usually nonspecific in nature and common to those of laparoscopic access. In our case history, 3 cases of internal bleeding (none of which were identified in GI transit) with formation of intra-abdominal meta-surgical haematomas were found, none of which showed active supply. The nature of these blood collections was probably attributable to a venous bud that spontaneously stopped with the increase in volume of the haematoma itself.

Overall, in the evaluation of the complications of the sleeve gastrectomy, GI transit plays a crucial role in the preliminary identification of the leaks, especially if they are larger, while it is not very sensitive both for small leaks, and for the evaluation of the extension of the patho- logical process, situations for which it is necessary to resort to the deepening TC. In no case has GI transit proved useful in identifying metasurgical peritoneal haematomas. Ten (14\%) post-operative complications of gastric bypass with Roux loop were found. Of these, 3 (30\%) were anastomotic dehiscences and 7 (70\%) were obstructive syndromes of the small intestine. Anastomotic dehiscences are early complications that usually affect the gastro-jejunal anastomosis $[10,18,19]$. The leaks can be small in size and self-limiting in paucisymptomatic patients, or they can assume considerable size with septic complications $[24,25]$. For this reason, an early diagnosis is indispensable, in which the GI transit plays a limited role due to its low sensitivity $[10,26,27]$. These data are confirmed in our case studies, because the diagnosis of leakage at the GI transit was possible in only 1 of the 3 cases, making it necessary to resort to CT to identify the spreading, any fluid collections, and free air.

Obstructive syndromes of the small intestine are relatively common complications after gastric bypass, with an incidence varying between 0.4 and $7.45 \%$ [15]. Among these, the most difficult to diagnose has been linked to the presence of an internal hernia. Internal hernia in the Petersen space is defined as the dislocation of the intestinal segment immediately adjacent to the packaging of the entero-enteric anastomosis through the mesentery of the anastomosed viscera. In the presence of a framework of frank occlusion, the CT signs of internal hernia, among which the presence of the "mesenteric swirl", i.e. the twisting of the fat and the mesenteric vessels around the root of the meso itself, should always be carefully researched $[26,27]$. In our experience, the diagnosis of the only case of internal hernia occurred during the surgery, because the CT examination had not highlighted the pathognomonic signs of internal hernia but only signs of marked suffering of the mesentery. This condition probably occurred both due to the early execution of the CT examination in relation to the onset of symptoms, and to the timely intervention of the surgeon. In this case $\mathrm{CT}$ proved to be a very specific method for the diagnosis of internal hernia on the basis of the characteristic signs, but it is very sensitive in placing the suspicion of complication on the basis of signs of intestinal suffering. An incorrect gastro-jejunal anastomosis, on the other hand, can be responsible for trans-hiatal herniation of the anastomosed gastric fundus, with anomalous over-distension and slowing of the transit of the food bolus in the channel assigned to its passage. The hiatal hernia highlighted in a patient, and involving both the gastroenteric anastomosis and the excluded gastric pocket, generally does not represent a surgical emergency; its treatment can only be considered based on the patient's clinical status. CT allows us not only to evaluate the intrathoracic site of the gastroenteric anastomosis, but above all it allows us to explore the excluded gastric pocket, which cannot be assessed by $\mathrm{X}$-ray or by endoscopy. 
As regards the other complications, all of a non-specific nature, such as the occlusions on the bridle, the flies, and the strangulated laparoceles, CT played a fundamental role in assessing the site of the obstruction and the planning of the intervention based on the degree of urgency of the disease. In particular, CT indicates with extreme precision the location of the volvulus, the torsion of its mesenteric vascular axis, and with evidence, at times, of signs of both arterial and venous wall ischaemia as well as of faecaloid stasis within the loop concerned [28-30]. In the case of laparocele, the CT is definitive, especially in the presence of multiple laparoceles, in identifying the herniated loop and evaluating the state of its walls that may be affected by infarct phenomena in the event of constriction. This study has some limitations: there was no direct comparison with a group of symptomatic-negative patients on GI and CT exams. Furthermore, the number of patients was small.

\section{Conclusions}

CT should be used to clarify all doubtful or clinically discordant GI transit exam results. The most frequent complications observed included obstructive syndrome of the small intestine (SBO, small bowel obstruction). The participation of a radiologist in the qualification and post-operative evaluation is important for bariatric surgery patients.

\section{Conflict of interest}

The authors report no conflict of interest.
Reference

1. MacDonald KG Jr. Overview of the epidemiology of obesity and the early history of procedures to remedy morbid obesity. Arch Surg 2003; 138: 357-360.

2. Must A, Spadano J, Coackley EH, et al. The disease burden associated with overweight and obesity. JAMA 1999; 282: 1523-1529.

3. Luca Busetto, Dror Dicker, Carmil Azran, et al. Practical Recommendations of the Obesity Management Task Force of the European Association for the Study of Obesity for the Post-Bariatric Surgery Medical Management Obes Facts 2017; 10: 597-632.

4. Nguyen NT, Varela JE. Bariatric surgery for obesity and metabolic disorders: state of the art. Nat Rev Gastroenterol Hepatol 2017; 14: 160-169.

5. Nguyen NT, Goldman C, Rosenquist CJ, et al. Laparoscopic versus open gastric bypass: a randomized study of outcomes, quality of life, and costs. Ann Surg 2001; 234: 279-289.

6. Puzziferri N, Austrheim-Smith IT, Wolfe BM, et al. Three-year follow-up of a prospective randomized trial comparing laparoscopic versus open gastric bypass. Ann Surg 2006; 243: 181-188.

7. Chandler RC, Srinivas G, Chintapalli KN, et al. Imaging in bariatric surgery: a guide to postsurgical anatomy and common complications. AJR Am J Roentgenol 2008; 190: 122-135.

8. Mingrone G, Panunzi S, De Gaetano A, et al. Bariatric-metabolic surgery versus conventional medical treatment in obese patients with type 2 diabetes: 5 year follow-up of an open-label, single-centre, randomised controlled trial. Lancet 2015; 386: 964-973.

9. Catelli A, Ponsiglione A, Capaldo I, et al. Giant endometriod ovarian cancer: the role of dagnostic imaging and figo staging. Euro Mediterranean Biomedical Journal 2020; 15: 94-96.

10. Konecki D, Grabowska-Derlatka L. Correlation between findings of multislice helical computed tomography (CT), endoscopic examinations, endovascular procedures, and surgery in patients with symptoms of acute gastrointestinal bleeding. Pol J Radiol 2017; 82: 676-684.

11. Corvino A, De Rosa D, Sbordone C, et al. Diastasis of rectus abdominis muscles: patterns of anatomical variation as demonstrated by ultrasound. Pol J Radiol 2019; 84: e542-e548.
12. Corvino A, Pignata S, Campanino MR, et al. Thyroglossal duct cysts and site-specific differential diagnoses: imaging findings with emphasis on ultrasound assessment. J Ultrasound 2020; 23: 139-149.

13. Levine MS, Carucci LR. Imaging of bariatric surgery: normal anatomy and postoperative complications. Radiology 2014; 270: 327-341.

14. Gaetke-Udager K, Wasnik AP, Kaza RK, et al. A guide to imaging in bariatric surgery. Emerg Radiol 2014; 21: 309-319.

15. Riaz RM, Myers DT, Williams TR. Multidetector CT imaging of bariatric surgical complications: a pictorial review. Abdom Radiol (NY) 2016; 41: 174-188.

16. Lim R, Beekley A, Johnson DC, et al. Early and late complications of bariatric operation. Trauma Surg Acute Care Open 2018; 3: e000219.

17. Pieroni S, Sommer EA, Hito R, et al. The "O" sign, a simple and helpful tool in the diagnosis of laparoscopic adjustable gastric band slippage. AJR Am J Roentgenol 2010; 195: 137-141.

18. Swenson DW, Pietryga JA, Grand DJ, et al. Gastric band slippage: a case-controlled study comparing new and old radiographic signs of this important surgical complication. AJR Am J Roentgenol 2014; 203: 10-16.

19. Mehanna MJ, Birjawi G, Hicham A, et al. Complications of adjustable gastric banding, a radiological pictorial review. AJR Am J Roentgenol 2006; 186: 522-534.

20. Blachar A, Blank A, Gavert N, et al. Laparoscopic adjustable gastric banding surgery for morbid obesity: imaging of normal anatomic features and postoperative gastrointestinal complications. AJR Am J Roentgenol 2007; 188: 472-479.

21. Nocca D, Frering V, Gallix B, et al. Migration of adjustable gastric banding from a cohort study of 4236 patients. Surg Endosc 2005; 19: 947-950.

22. Chivot C, Robert B, Lafaye N, et al. Laparoscopic sleeve gastrectomy: imaging of normal anatomic features and postoperative gastrointestinal complications. Diagn Interv Imaging 2013; 94: 823-834.

23. Ekinci T, Stein MW, Mazzariol FS, Wolf EL. Laparoscopic sleeve gastrectomy: everything the radiologist needs to know. Clin Imaging 2017; 43: 36-41. 
24. Pernar LI, Lockridge R, McCormack C, et al. An effort to develop an algorithm to target abdominal CT scans for patients after gastric by-pass. Obes Surg 2016; 26: 2543-2546.

25. Blachar A, Federle MP, Pealer KM, et al. Gastrointestinal complications of laparoscopic Roux-en-Y gastric bypass surgery: clinical and imaging findings. Radiology 2002; 223: 625-632.

26. Dilauro M, McInnes MD, Schieda N, et al. Internal hernia after laparoscopic Roux-en-Y gastric bypass: optimal CT signs for diagnosis and clinical decision making. Radiology 2017; 282: 752-760.

27. Lockhart ME, Tessler FN, Canon CL, et al. Internal hernia after gastric bypass: sensitivity and specificity of seven CT signs with surgical correlation and controls. AJR Am J Roentgenol 2007; 188 : 745-750.
28. Karila-Cohen P, Cuccioli F, Tammaro P, et al. Contribution of computed tomographic imaging to the management of acute abdominal pain after gastric bypass: correlation between radiological and surgical findings. Obes Surg 2017; 27: 1961-1972.

29. Corvino A, Setola SV, Sandomenico F, et al. Synchronous tumours detected during cancer patient staging: prevalence and patterns of occurrence in multidetector computed tomography. Pol J Radiol 2020; 85: e261-e270.

30. Corvino A, Catalano O, de Magistris G, et al. Usefulness of doppler techniques in the diagnosis of peripheral iatrogenic pseudoaneurysms secondary to minimally invasive interventional and surgical procedures: imaging findings and diagnostic performance study. J Ultrasound 2020; 23: 563-573. 